\title{
A comparative study of Lean implementation in higher and further education institutions in the UK
}

\author{
Andrew J. Thomas \\ Business School, \\ University of Glamorgan, Pontypridd, UK \\ Jiju Antony \\ School of Management and Languages, \\ Heriot-Watt University, Edinburgh, UK \\ Mark Francis \\ Business School, Cardiff Metropolitan University, \\ Cardiff, UK, and \\ Ron Fisher \\ Griffith University, Queensland, Australia
}

\begin{abstract}
Purpose - The purpose of this paper is to investigate and understand the differences that exist between educational institutions in the methods and practices employed in the development and implementation of Lean projects. Whilst many Higher Education Institutions (HEIs) are now starting their journey towards effectively implementing Lean, Further Education Institutions (FEIs) have treaded this well worn path many years previously and so the aim of this paper is to find what key features and issues FEIs have put in place to assist them in implementing Lean projects and whether HEIs can learn from such institutions.

Design/methodology/approach - The paper applies two research methods in an attempt to understand the differences between the institutions and hence understand the key features that can be used to better implement Lean initiatives. First, through a series of focus groups, the authors employ a low-level form of Group Consensus Theory in an attempt to understand the organisational dynamics surrounding the adoption of Lean. This is used to understand whether Lean improvement teams within FEIs implement such initiatives with the autonomy and support from senior management. Second, the same group members, detailed interviews were held in order to identify further and more specific information around the tools and techniques employed in the implementation of Lean initiatives in both HEI and FEIs.

Findings - The study found that although FEIs had much more experience in the design, development and implementation of Lean initiatives, the organisational infrastructure and dynamics towards driving Lean in FEIs was less well embedded in to the culture of the respective institutions than first thought and, that Lean had been developed and driven initially by a consultancy-based approach and around a tool-driven mentality. It was seen that whilst HEIs were generally slower in getting off the mark, there seemed to be more enthusiasm and willingness to drive such initiatives forward and in a more systematic and holistic manner even though some of the projects were in their very early stages of implementation.

Research limitations/implications - Whilst this work provides key information on how Lean initiatives are implemented across different institution types, the work has only looked at a very small sample of two teaching focused HEI and two FEIs. The work will need to be extended much more widely to incorporate a larger set of HEIs (both research and teaching focused) in order to provide a more complete map of Lean development in HEIs.
\end{abstract}


Practical implications - The aim of the paper is to provide Lean project leaders in HEIs with some additional key insights towards the cultural and organisation dynamics that exist in educational institutions other than HEIs in order to assist them in developing further and more comprehensive Lean programmes.

Originality/value - This paper is the first of its kind to study the organisational and cultural dynamics that exist between differing educational institutions in their approaches towards the

implementation of Lean and business improvement programmes. The key features highlighted in this

work raise important issues regarding the need and importance of developing team dynamics around project implementation.

Keywords Lean, Higher education, Further

education

Paper type

Research paper

\section{Introduction}

The higher education sector in the UK is changing rapidly. The current economic crisis as well as the change in the way Higher Education Institutions (HEIs) receive funding from their respective Higher Education Funding Councils has created a major shift in emphasis. Through changes in governmental policy relating to university fees, students now hold a major chunk of the income stream that HEIs so badly need. Therefore, in order to remain competitive in the face of greater competition for student places, HEIs are focused on providing more competitive offerings to their students. It is not likely that anyone can predict in what direction HE funding will go over the next few years (Barber et al., 2013) but one thing is certain, HEIs will need to do more with less, develop new teaching and learning strategies, differentiate by being distinct in the products and services it offers, offer a greater value adding proposition to the student and continue to be more "customer" focused. Therefore, in order to achieve the above aims, HEIs will need to become Leaner and more efficient in what they do and this will inevitably call on them to adopt improvement strategies such as Lean to assist in their efforts to achieve a more efficient and productive institution that is economically sound.

The implementation of business improvement strategies such as Lean, Six Sigma, etc. brings its own challenges. Antony et al. (2012) identify a number of key issues in the implementation of Lean Six Sigma in HEIs. Their work outlines that the strategy of achieving "leanness" is not clear to many senior managers and this is primarily due to: the lack of awareness of the benefits of Lean in non-manufacturing industry; a lack of commitment and support from senior executive teams makes it difficult to foster a culture of continuous improvement; a lack of process thinking and process ownership since process thinking is not at all prevalent in many HEIs and, establishing processes at the work place requires a change of mindset. A lack of visionary leadership is a fundamental barrier in the successful introduction and deployment of such initiatives according to Snee (2010) and, the culture prevalent within HEIs limits the opportunity for staff to feel that they are part of the organisation and to openly talk about their improvement suggestions. Antony et al. (2012) also identified a lack of communication at various levels across HEIs along with a lack of resources (time, budget, etc.) and the weak linkages that exist between continuous improvement projects and the strategic objectives of the HEIs as being major inhibitors to effective Lean implementation.

On the other hand, however, although many challenges and barriers exist, the benefits can be many. Radnor and Bucci (2011) identify a positive set of issues resulting from Lean implementation. These include: positive changes in the culture in specific areas of the HEIs where staff have understood the concept of Lean and realise that Lean has given them the capabilities to enable them to arrive at effective solutions; the empowerment of staff thus enabling them to make key decisions and enact changes 
to their business processes; enabling HEIs to tackle the quick wins and create immediate impacts, which free up capacity to enable staff to look at improving other processes; gives an opportunity for staff to be involved in process improvement activities that are focused on internal processes.

On the general issue of Lean implementation in HEIs, there is now an increasing volume of academic literature that is now emerging in the area. Table I provides an overview of the key Lean literatures applied to HEIs. The literature around the application of Lean in HEIs is still very much in its infancy compared to the plethora of information from Lean in manufacturing. Generally, little literature exists around the organisational dynamics and knowledge management aspects of implementing Lean in HEIs whereas, very little academic literature at all exists in the application of Lean in Further Education Institutions (FEIs). Therefore, this paper fills this gap in knowledge.

Over the past decade or so FEIs have had to cope with increased competition and reduced student funding as well as the fragmentation of funding streams towards demand led funding (Leitch Review of Skills, 2006). Therefore, FEIs have had to embark on the Lean journey much earlier in order to remain competitive and have had to overcome the challenges that are now being experienced by HEIs. To identify the essential differences between HEIs and FEIs, the reader is directed to the Further and Higher Education Act (The Stationary Office, 1992). However, whilst there are key differences between HEIs and FEIs in terms of their focus and purpose, the same fundamental constraints exist for both, namely; reduced funding, increased competition and the need to ensure student experience and quality of delivery is continuously improved. This paper will therefore compare HEI Lean initiatives with those that are employed in the FE sector to see what the key similarities and differences are and, what can be learnt from the application of Lean thinking in this closely aligned area of operation. Focus groups (FGs) made up of two FEIs and two HEIs are used to develop the research programme.

Prior to the start of the survey and after obtaining agreement off the institutions to undertake this survey, the authors undertook a detailed observation of the Lean activities being purported to be undertaken in each institution. Both FEIs had been on their respective Lean journeys for over ten years and implemented a wide range of Lean tools and techniques. Both institutions applied Value Stream Mapping, waste identification and 5S techniques and employed a range of statistical data to measure systems performance and improvement. Other tools and techniques observed included; Six Sigma application predominantly around the use of the DMAIC approach and maintenance techniques adopted around equipment servicing with a focus on systems availability and uptime. However, the key to increased performance was seen through maximising human and technology resources and ensuring that all resources were operating at their design intent (staff teaching to contracted hours, focus on commercial income generation in nonteaching time, etc.).

HEIs were starting some distance behind the FEIs with both institutions approximately four years in to their Lean implementation journeys. As a where the application of specific tools and techniques were less evident. Most Lean projects were focused upon improving support functions such as finance and admissions departments. Asset management was less robust but there was evidence of process mapping and a drive towards ensuring staff were working to their design intent (although this was better managed in HEI 1 than in HEI 2). 
Comm and Questionnaire to 18 public and private universities Mathasisel in the USA and analysed

(2005a)

Hines and

Lethbridge (2008)

Semi structures interviews with client universities in the USA along with a comprehensive literature review to provide an understanding of various Lean university initiatives

Comm and Mathasisel (2005b)

An open-ended qualitative questionnaire was developed, administered to 18 public and private university representatives and analysed

Emiliani (2004) Case study development of Lean implementation in to a graduate-level Business Studies course in a US university

Antony et al. A viewpoint paper on critically evaluating the (2012) effectiveness of Lean Six Sigma in HEIs

Antony (2014) A theoretical paper based on existing literature and authors' experiences in the HE sector in to the readiness factors (RFs) around CI initiative introduction

Doman (2011) A case study approach to demonstrate that Lean principles and practices utilised in industry can be successfully applied to improve higher education administrative processes
The paper considers the issue of sustainability within US universities and describes the role that Lean implementation plays in achieving economic sustainability

Although the universities surveyed implemented Lean often without knowledge that they were implementing "lean" practices, their application has often reduced waste, improved operational efficiency and contributed to sustainability

Early attempts to develop a Lean university system There is much potential to improve customer value focused almost entirely on maintaining the technical and eliminate waste in universities. However, their thinking around Lean in manufacturing. This often study outlines that it is increasingly evident that the meant that universities failed to fully implement Lean correctly and effectiveness was lost

The participants at these surveyed universities shared with the interviewers the institutional Lean "best practices" that they feel will contribute to the sustainability of their universities

The case study highlights the typical tools, techniques and systems employed in the actual delivery of the course. Most tools employed are based around Lean manufacturing principles

Through the authors experiences of implementing Lean Six Sigma, the effectiveness of LSS is made and barriers including the importance of strategic barriers and challenges of implementing LSS is made alignment of LSS projects with HEI vision,

The paper identifies RFs which are essential for the RFs identified from this work include; effective

implementation and sustainability of LSS. RFs are leadership and vision, management commitment required prior to embarking on Lean, LSS, etc.

The paper outlines a structured approach to enabling students to undertake a Lean implementation process. The paper shows that through good guidance and support, effective Lean improvements can be made communication effectiveness, etc.

academic environment is harder to change than many conventional Lean environments. In common with many older universities, the strategic structures unaccustomed to rapid change Best practices identified include; strong financial ffective training, effective decision making around course closure/survivability

Evaluation made of application of Lean tools such as; 5S, continuous improvement, JIT in script marking, etc. Positive improvements seen in student experience and instructor performance customer focus, right people

An outline framework is identified and the work is focused upon a support function within the university control, effective top-down direction and leadership, and resources, linking CI to university strategy,

(1) 


\section{Research method}

The aim of this research programme is to initially identify the key management strategies employed within both FEIs and HEIs in order to determine the effective implementation of Lean in such institutions. Furthermore, the authors are particularly interested in understanding the issues around the organisational dynamics and the acquisition, integration and application of learning and knowledge about Lean implementation in order to sustain Lean implementation projects in HEIs.

In order to fully investigate both the "organisational dynamics/knowledge acquisition and management" issue and, the "management strategies" issue, a two-phase programme of research is undertaken. First, through FG development, a practical approach to investigating and characterising the dynamics of learning and implementing Lean initiatives amongst FEIs and HEIs is undertaken through adopting the Group Consensus Theory (GCT). Second, through face-to-face interviews and questionnaire feedback, a detailed study and analysis is undertaken to determine the types of Lean strategies undertaken from Lean implementation in both HEIs and FEIs. The study in to the organisational dynamics and knowledge management is shown next.

\subsection{Organisational dynamics around Lean implementation}

There is an increasing amount of empirical research that supports the fact that GCT has been successful in reducing communication barriers and facilitating decision-making activities by increasing participation and providing increased opportunities to influence the opinions of others in groups (Fjermestad, 2004; Read et al., 2012). Group decision support has also been developed to help identify the core knowledge to be developed by an organisation (Lin et al., 2007). However, there is little evidence that demonstrates the usefulness of GCT as a tool to support business process improvement in HEIs. The approach to characterising organisational dynamics which is described in this paper is based on a form of "group inquiry" as outlined by Read et al. (2012). This paper presents the authors experience of extending the "group inquiry" concept in to both FEIs and HEIs. Features of this work as outlined by Read et al. (2012) and applied here are:

- characterising the group dynamics and group learning around Lean implementation in FEIs and HEIs where there have been few previous empirical studies; and

- a field-study on how this form of group analysis can aid business improvement teams to access their own knowledge and share best practice.

The method is under-pinned by a social perspective of the process of organisational learning, focusing on the experience and validation abilities of groups of people in a work place (Higgins, 2009; Read et al., 2012). From this perspective learning emerges from the interactions of the group and, learning itself is concerned with characterising and defining local knowledge about given business improvement practices. A detailed application of the technique is now outlined.

\section{Research study - Stage 1}

In order to ensure the workshops and groups were of a manageable size, the authors undertook two workshops and two FG studies with the participants and institutions listed in Table II. In Workshop 1 and FG 1, participants made up of FEI 1 and HEI 1 attended. Likewise, in Workshop 2 and FG2, participants from 
HEI 2 and FEI 2 attended. Selection of which HEI and FEIs to attend the workshops was undertaken randomly.

Prior to the workshops and FGs, the authors reviewed the question sets provided from the work of Read et al. in order to contextualise the questions and to reduce the ambiguity surrounding what was actually being asked in each question. Since the question sets were generic in nature and were focused upon understanding the organisational and group learning dynamics around the application of Lean in the respective institutions, there was not a need to reduce the number of questions but, to just contextualise the questions somewhat so that a common understanding amongst all participants was achieved.

The HEIs included in the study were UK based and expressed a preparedness to take part. These HEIs are considered "post 92" academic institutions and were primarily teaching focused. The FEIs were selected based on their preparedness to take part in the survey. The FEIs by their very nature are teaching institutions.

Focus and workshop group sizes were quite large at approximately 25 participants each. Each session involved a mix of staff at all levels from within the institutions. Two stated aims were given to each participant within the groups before staring the workshop and FG. These were:

- to generate collective reflection on how the institutions lead and manage Lean project implementation; and

- to initiate a dialogue on how the institutions are led and managed more generally in order to identify any key cultural and/or organisational behaviour issues that would make the respective institutions more or less capable of implementing Lean projects.

The workshop session lasted one hour on average and this consisted of the authors outlining the rationale for the study, establishing the aims and objectives of the work and, introducing the participants to the questions. The FG was then run immediately after the workshop. Each FG session lasted approximately two hours, and was facilitated by a facilitator, who was not one of the authors in order to limit bias and intervention. The facilitator was asked to stay out of the dialogue as much as possible, with the exception of occasions when it was potentially insightful to ask participants to provide an example to illustrate the point they were making. A member of the author team observed each session but did not participate or contribute in any of the sessions.

The members of the FG entered their personal response to each of a series of questions that were presented on a screen, without any discussion or feedback. The question set consisting of 30 questions was used and was designed around three key issues which are centrally concerned with organisational learning, following a theoretical framework presented by Vince (2001) and developed by Read et al. (2012). The questions were designed using a five-point Likert-type, agree/disagree scale.

\begin{tabular}{|c|c|c|c|c|c|}
\hline Participants & FEI 1 & FEI 2 & HEI 1 & HEI 2 & \\
\hline Deans/faculty directors & 2 & 2 & 3 & 2 & Table II. \\
\hline Faculty/school head & 4 & 3 & 4 & 3 & Number and \\
\hline Department head & 4 & 3 & 4 & 4 & type of participant \\
\hline Lecturer/senior lecturer & 2 & 2 & 2 & 2 & involved in study \\
\hline
\end{tabular}


The question set is shown in Table I and as stated previously, is taken from the work of Read et al. (2012) in which they employed a similar study in a manufacturing organisation to investigate situated knowledge acquisition and development. The questions have direct applicability to our study and so have not been changed or modified although the context for each question has been changed slightly to focus upon Lean project implementation.

The outputs from the FG sessions were recorded for analysis in two ways. The inputs from the questionnaires provided a quantitative record of all judgements. The quantitative information provided by the FG gave an understanding of the feelings and opinions from the groups whereas, the ensuing dialogue and discussion provided insights into the reasons why these views were held. Table II shows the list of questions which were answered by each participant in each FG.

Each member of the FG marked each question using the Likert scale. All participants were requested to provide their opinions for each question asked. Once complete, the facilitator collected the paper responses. The authors then fed the information into a spreadsheet and then fed the aggregated score to the FG for further discussion. This enabled the FG members to have a level of anonymity around their personal response and, the FG were then able to discuss the aggregate scores rather than focusing upon individual scores.

During the FG session the aggregate feedback score to each question was used as a means of stimulating a conversation focused on the range of responses (i.e. range of perceptions). A low-level form of facilitation, which simply summarised each display, was found sufficient to generate conversations focused on the reasons for differences. It was found that individuals sometimes declared their own input, and sometimes preferred to maintain their anonymity. The particular usefulness of the bar charts to display the aggregate score to each question maintained a collective focus. The discussions that emerged from responses to the question set were transcribed to provide the researchers with insights into situated knowledge within each institution. Table III shows the question sets and also the scores obtained from both the HEI and FEI members within the FGs.

\subsection{Results of the FG work}

Different themes emerged from an analysis of the conversations with each FG. Whilst Figure 1 shows the responses to all 30 questions, for the purpose of this paper, the authors will only analyse the answers to the questions in which major disparities between the marks given to each question were seen. The aggregated results from four separately managed educational institutions (two HEIs and two FEIs) are shown in Figure 1.

\subsection{Feedback responses}

As can be seen, there were in some cases significant differences in the scores awarded by the FEIs and HEIs in terms of the responses obtained from each of these participant groups. These differences are demonstrated with reference to the four questions 2, 7, 22, 26 and 29. A brief analysis of the results is now undertaken.

Q2. The predominant management style is participative rather than "top-down". A marked difference in the scores was seen between HEI and FEIs. HEI members felt that the approach to Lean implementation was much more participative whereas FEI members felt that the initiatives had been driven down from senior management via 


\begin{tabular}{rlll}
\hline 1 & Decision-making power is widely shared throughout the organisation & 4 & 3.8 \\
2 & The predominant management style is participative rather than & & \\
& "top-down" & 3.8 & 2 \\
3 & I feel able to participate in decisions which are important to the & 3.7 & 3 \\
& organisation & 4 & 4 \\
4 & Senior managers commonly delegate decision making to others & 4 & 4.2 \\
5 & It is easy for me to raise awkward issues with managers & 3.5 & 2.5 \\
6 & Staff are encouraged to speak out & 4 & 2 \\
7 & Difficult issues are not normally brushed under the carpet & & \\
8 & Staff are "open" in their communications (e.g. there are few "hidden & 3.5 & 2.5 \\
& agendas") & 4.2 & 3 \\
9 & When the going gets tough, people help each other & 3.5 & 3.6 \\
10 & People are willing to admit when they make mistakes or errors of & 3 & 3.1 \\
& judgement & 3 & 4 \\
11 & Conflicts and disagreements are dealt with openly & 3.6 & 3.2 \\
12 & There is generally a high level of trust between members of staff & 3.1 & 3.4 \\
13 & I feel comfortable expressing my feelings in the organisation & 2.6 & 3.2 \\
15 & Important issues for the organisation are discussed readily & 2.8 & 2.1 \\
16 & Staff rarely feel anxious & 2.6 & 1.5 \\
17 & Staff rarely behave defensively & 3 & 2 \\
18 & We do not have a blame culture in the organisation & 3.2 & 4 \\
19 & Staff are encouraged to reflect on the organisation's processes & 4 & 3 \\
20 & I rarely feel threatened when things are going wrong & 3.5 & 4.5 \\
21 & I rarely feel powerless in the organisation & 3 & 4.5 \\
22 & Important issues are not avoided & 3 & 2.5 \\
23 & We have regular meetings to review progress and discuss issues & 5 & 3 \\
24 & I would welcome more responsibility within my job & 4.6 & 3.2 \\
25 & I am satisfied with the way I am managed by my immediate boss & 4.5 & 2.5 \\
26 & We learn from mistakes, and are not blamed for them & 4 & 3 \\
28 & I enjoy my work & 4 & 3 \\
& I trust the information I get from senior management & 4.2 & 1.5 \\
I receive open and honest feedback about my performance & 5 & 5 \\
stage of research \\
\hline
\end{tabular}

consultants. However, it must be noted here that FEIs were in the main, further down the route of Lean implementation at the same point in their implementation cycle when compared to HEIs. It was suggested from the FG that a more participative style has led to a lack of emergence of a leader who will drive various initiatives forward in the HEIs.

Q7. Difficult issues are not normally brushed under the carpet.

It was widely considered by the HEI respondents that the senior management teams tackled the serious issues relating to financial sustainability and the need to implement Lean initiatives "head on" whereas, FEI respondents felt that the difficult issues of financial sustainability were normally kept away from them so they were less understanding of the key sustainability agenda. However, when it came to tackling the key issue of applying Lean principles in to the management of teaching and learning, FEIs saw this as the central plank of their work whereas HEIs were implementing Lean 
Figure 1. Institutional comparison of marks given to each GCT question

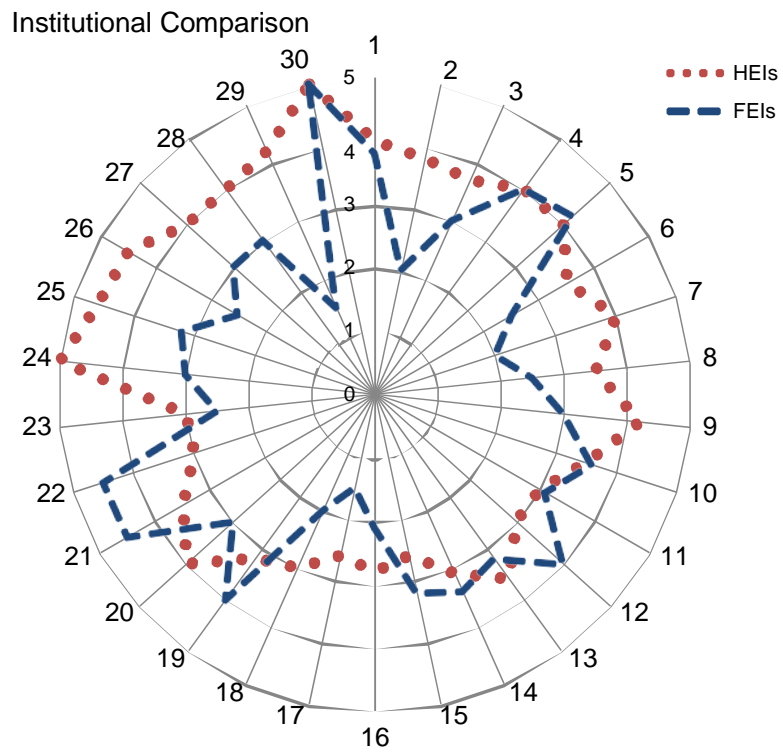

thinking in to the support systems such as admissions and finance. There seemed to be a much greater difference in focus between the two institutions.

Q22. We have regular meetings to review progress and discuss issues.

In HEIs, 70 per cent of participants stated that regular meetings are held to review progress and discuss issues, compared with 44 per cent from FEIs. Further probing as to the reasons why this was the case found that the opportunities for teaching teams in FEIs to meet on a regular basis was much less than in HEIs due to their significantly greater teaching workloads.

Q26. I enjoy my work.

It was noted that participants from HEIs responded much better to the question than those situated in FEIs. Discarding the usual pressures around students, etc., participants in HEIs felt they has greater autonomy to develop new opportunities and implement new ideas. However, on analysing the work being undertaken in these HEIs regarding new initiatives and the development of key Lean projects, none of the HEIs had developed anything of any note.

Q29. I have the opportunity to influence the way I work.

This question again scored highly with the HEI participants and in some ways, validates the observation seen in Q26. HEI participants were seen to have greater autonomy to influence the way they worked but, had not developed anything of particular note during the last four years of Lean development within their institutions.

Through discussion with the participants undertaking the survey, further richer contextualised information emerged that enabled the authors to undertake a deeper analysis of the key organisational issues surrounding Lean implementation. In general, FEI participants felt that there is strong leadership present in driving improvement initiatives and these initiatives seem to be focused around reducing waste from the value adding processes (teaching and learning). However, the means and approach 
towards driving change is seen as somewhat dictatorial and does not create the correct environment for driving harmonious change in the sector. Issues around workloads further prevent FEI programme development but senior managers outline the need for fast and focused change and improvement due to the workload pressures faced within the sector.

HEI participants, however, paint an improved picture where autonomy and freedom to work as part of a cohesive and well-structured team is available to them. Issues around work-loading and integration of projects in to current research and teaching is less of an issue. However, when comparing a HEIs progress towards Lean implementation at the same point in time as a FEIs, HEIs do not have seemed to move on at all tending to work around the edges of business processes and not implementing any real large scale improvement project. It seems that such institutions lack the leadership to drive such initiatives forward at present. Therefore, in summary the key issues identified from this phase of the research study are:

- waste focus and financial control;

- strategy formulation and deployment;

- customer focus;

- leadership; and

- people empowerment.

The key issues identified above are now used as the basis to further explore and compare the dynamics around how Lean is implemented in the FEIs and HEIs.

\section{Research study - Stage 2}

The second stage of the study was to identify and compare the key Lean implementation programmes being adopted within FEIs and HEIs. In this study, the participants remained the same as in the first research study and each institution was visited by a member of the research team. The FGs were re-established and the participants interviewed as part of the FG. Semi-structured questionnaires were used and an unstructured interview approach was undertaken to where elaboration on a particular answer was sought. Alongside the interview/questionnaire format, the authors observed the Lean implementation plans and probed the FGs where possible in order to obtain further information about the initiatives being employed and their level of progress.

As a means to providing focus and to identifying the typical questions that were required to be asked, the authors employed the results of the work undertaken in research study 1 as the basis in which to develop the questionnaire. In addition, the authors also included a further four Lean perspectives which were aimed at understanding the operational issues surrounding Lean implementation in the institutions. These additional questions were aimed at specifically focusing in on the Lean implementation elements of the initiatives exploring the specific leadership skills, tools employed, aspirations and the journey ahead whereas, the questions generated from the previous survey specifically looked at the organisation's approach to culture and empowerment in order to see if the Lean implementation was actually aligned to the institution's growth strategy. Table IV outlines the key issues obtained from the survey. Further discussion on these results is made in the next section. 
Business

elements

Waste focus strategy

(t)

deployment

Leadership

Culture and empowerment development and stifles innovation. SMT run a number of initiatives but fail to change the culture towards one of self-leadership and cultural change Use of virtual learning environments are key to efficient delivery

Highly regulated financial process with budgets to faculties and departments limited to operational issues only. Financial decision making is top-down with limited opportunity to use budget for anything other than consumable spend A clear vision that was focused on ensuring course sustainability and unending improvement of the student experience. Significant effort used in integrating courses at module level in order to ensure module enrolments were high. Lean focus on standardisation and maximising the value of human assets. Goes to the heart of the $\mathrm{T}+\mathrm{L}$ function

The FEI with a well established strategy showed evidence of deployments to the departments with faculty strategies being available and understood by departmental heads. Some deployment seen in the other HEI but this was patchy and no real staff buy-in observed

At lecturer level, focus is primarily on the studen on Inspectorate financial issues (Antony et al., 2012).

Leadership is very much "top-down" in nature at college director level. Early stages of implementation driven through consultants
Poor. Hierarchical approach limits cultural

Higher Education Institutions

Lean initiatives tend towards reducing waste from support functions. Leads to isolated and unconnected Lean thinking with limited impact. Neither institute tackled the value adding area of teaching and learning in order to drive change. VLE used alongside virtual conferencing systems to deliver simultaneous lecturing on multi-campus in order to reduce multiple lecturing points

Held at faculty level through Dean. Local decisions made quicker. Budgets enable staff employment and support of Lean initiatives

Lean strategy less focused in general with a wider strategic aim that is somewhat less clear to implement due to its wider ranging nature. Lean focus not fully on $\mathrm{T}+\mathrm{L}$

function but support services were being improved before the $\mathrm{T}+\mathrm{L}$ function is tackled

Deployed effectively down to the staff via the Faculty Dean. Local strategies encouraged so staff feel empowered

At lecturer level, focus is totally on the student experience. At senior management level, focus is on quality and financial issues

Leadership devolved through schools who have responsibility for Lean implementation. Lean teams tend to be more self-leading and more dynamic

Good. Staff empowered to undertake local initiatives but focus is on income generation rather than process efficiency and improvement

\section{Further Lean perspectives}

Lean leadership Driven from the top. Deputy Principal given job to drive Lean initiatives. Both FEIs initially

Unknown by staff. No real work undertaken on what Lean is and its impact on university. employed consultants but felt they did not tackle the differences in education well enough. Went on own initiatives and journey. Have not called their programmes Lean.
SMT know they need to improve but have limited understanding of where to go and what to do. Using consultants to drive VSMs at present. No recognition system employed
Table IV.

Key findings from research programme 


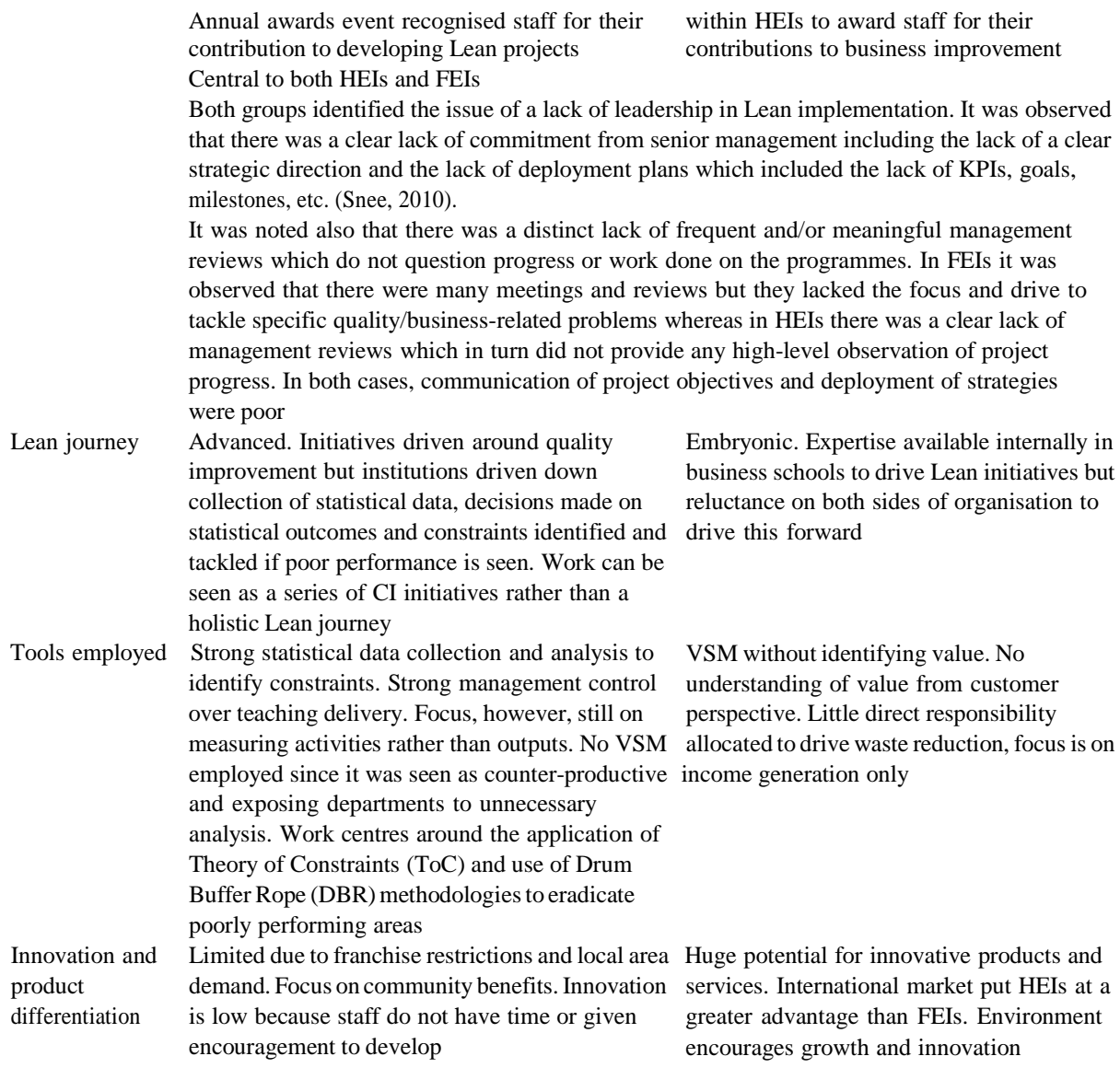

Tools employed Strong statistical data collection and analysis to identify constraints. Strong management control over teaching delivery. Focus, however, still on measuring activities rather than outputs. No VSM employed since it was seen as counter-productive and exposing departments to unnecessary analysis. Work centres around the application of Theory of Constraints (ToC) and use of Drum Buffer Rope (DBR) methodologies to eradicate poorly performing areas

Innovation and Limited due to franchise restrictions and local area product differentiation

Embryonic. Expertise available internally in business schools to drive Lean initiatives but reluctance on both sides of organisation to drive this forward

VSM without identifying value. No understanding of value from customer perspective. Little direct responsibility allocated to drive waste reduction, focus is on income generation only demand. Focus on community benefits. Innovation is low because staff do not have time or given encouragement to develop

Huge potential for innovative products and services. International market put HEIs at a greater advantage than FEIs. Environment encourages growth and innovation

\section{Discussion and conclusions}

Research study 1 outlined that HEIs were more likely to develop holistic strategies to implementing Lean due to them having improved infrastructural capacity (time, support, etc.), research study 2, however, identified that FEIs had greater experience and knowledge of developing and implementing business process improvement programmes than HEIs although a significant level of "project fatigue" had set in to the teams and the motivation towards future developments looked less obvious. Given the nature and positioning of FEIs also, the opportunities to differentiate products and tackle international markets is limited therefore it could be argued that product diversity and complexity is lower thus enabling Lean initiatives to be implemented on a more stable product platform.

However, what was a key finding in this work was that FEIs implemented Lean initiatives in a focused attempt to reduce operating costs whilst returning a 
better student/learner experience. Therefore it was possible to see the rationale for implementing such improvement programmes. Asking the same question of HEIs, however, the implementation of Lean with a clear focus on improving the bottom line was not readily seen. This could be because HEIs are only starting to wake up to the idea that efficiency savings are required in the future and that Lean implementation will play an increasing role in the modern university.

Both FEIs used consultancy companies in the past to help initiate programmes but had dropped the idea for favour of going it alone after the initial understanding of Lean was delivered to its staff. The FEIs now both run their own Lean implementation programmes at it was observed that some rudimentary application of the Theory of Constraints (ToC) (Goldratt, 1997) and Six Sigma (Snee and Hoerl, 2002) was being employed in some areas. This saw the institutions using more focused tool-driven approach with the focus on improving student experience whilst reducing waste and cost. At an infrastructural level, the FEIs costs and expenditure is highly controlled at senior management level. However, the command and control mentality spills over in to the management of staff. This results in a lack of motivation which impacts on the institution's ability to innovate and find solutions to various efficiency-based problems. Although, there is greater use of shared services and a definite focus on maximising the value of both the human and infrastructural assets of the organisation.

The HEIs observed were starting to develop a culture of Lean but had failed in the past four years to do anything of any significant impact. The issue of tackling support functions with prescribed methods of Lean often hinders growth and makes little impact to the overall programme (Comm and Mathaisel, 2005a). Tackling the teaching and learning area is key for FEIs but seems to be an area that is not focused upon yet by the HEIs. Within the HEIs also, there seems to be a focus on making sure the Lean process is seen to be done correctly and that the Lean process is aesthetically correct (training rather than doing) rather than doing the core work needed to drive change in to the organisations (Snee, 2010). FEIs on the other hand were focused more on using the respective tools and techniques as starting points and then developed the techniques in to a usable format through active implementation. This often meant that the application of tools and techniques were fairly rudimentary in nature and frequently were not called by their formal names (ToC for instance). However, the institutions observed in this paper seem to have the correct attitude and cultural mindset amongst the staff to succeed but seem to lack the necessary leadership skills or focus required to drive change. This may be because the full impact of governmental cuts and student fees have not yet driven the need for HEIs to adopt seriously the Lean philosophy.

Whilst the survey work identifies clear weaknesses in the HEI approach to implementing Lean, FEIs also suffer from a number of key issues. In general FEIs seem to employ a "tool oriented" approach to Lean implementation and as a result, implementation is somewhat piecemeal and incoherent in some cases. There seems to be a lack of a clear approach to what Lean means and so, institutions are driving Lean through a cost down mentality rather than a "value add" focus. Discussions with senior managers suggests that they are aware of this issue but outline the problems associated with a lack of clear time to implement such activities since teaching and lack of resources are forcing these colleges to apply a Lean technique or tool at to achieve a "quick win" as a result of the exercise. Therefore, it can be seen that many Lean initiatives are being undertaken but there is little overarching philosophy or strategy being employed to manage the process of Lean implementation. However, 
HEIs tended to try and implement a "pure" version of Lean and as a result, did not seem to make the gains required and the improvements in performance that were seen by the FEIs.

This research project has only started to make inroads in to understanding the culture and dynamics of Lean implementation in HEIs. Whilst we are able to learn key lessons from allied industries and sectors, what seems to be clearly lacking from within FEIs is the appetite and leadership required to drive Lean initiatives in a coherent and value adding way. However, HEIs also need clear direction and leadership in ensuring the jobs gets done since many projects are shown less than adequate levels of progress and return on investment and capital employed.

The survey identified that HEIs have the capacity and infrastructure to develop a business improvement mindset and so this needs to capitalised upon. However, the authors are mindful that only two HEIs were analysed in this paper and that there is possibly a wealth of good practice within HEIs throughout the UK. Therefore, future research should focus on developing a landscape map of where the various Lean initiatives are being developed throughout the HE sector in the UK and, to start to piece together a coherent approach to developing a purpose built Lean operating model. The authors are currently undertaking a longitudinal study in to mapping the Lean initiatives in HEIs and this will be the focus of future academic outputs.

The aim of the paper is to provide Lean project leaders in HEIs with additional key insights in the area of cultural and organisation dynamics that exist in educational institutions other than those seen in HEIs so as to assist HEIs in developing further and more comprehensive Lean implementation programmes. In order to do so, this paper offers Lean practitioners and academics an insight in to the practical applications of Lean in both HEIs and FEIs and, to provide a focus for future Lean implementation and to also act as a mechanism in which Lean practitioners can measure and compare where they are on their respective Lean journeys. The authors are seeking further information on Lean implementation programmes undertaken elsewhere and so it is hoped that this work will provide the basis for further development of ideas and stimulate the application of Lean in other academic institutions where future collaborations will be developed.

From a methodological perspective, the use of the low-level GCT framework for identifying the basic organisational dynamics of the institutions was seen as an effective technique for information acquisition and analysis.

\section{References}

Antony, J. (2014), "Readiness factors for the Lean Six Sigma journey in the higher education sector", International Journal of Productivity and Performance Management, Vol. 63 No. 2, pp. 257-264.

Antony, J., Krishan, N., Cullen, D. and Kumar, M. (2012), "Lean Six Sigma for higher education institutions (HEIs): challenges, barriers, success factors, tools/techniques", International Journal of Productivity and Performance Management, Vol. 61 No. 8, pp. 940-948.

Barber, M., Donnelly, K. and Rivizi, S. (2013), An Avalanch is Coming - Higher Education and the Revolution Ahead, Institute of Public Policy Research Publication, London.

Comm, C.L. and Mathasisel, D.F.X. (2005a), "A case study in applying Lean sustainability concepts to universities", International Journal of Sustainability in Higher Education, Vol. 6 No. 2, pp. 134-146. 
Comm, C.L. and Mathasisel, D.F.X. (2005b), "An exploratory study of best Lean sustainability practices in higher education", Quality Assurance in Education, Vol. 13 No. 3, pp. 227-240.

Doman, M.S. (2011), "A new Lean paradigm in higher education: a case study", Quality Assurance in Education, Vol. 19 No. 3, pp. 248-262.

Emiliani, M.L. (2004), "Improving business school courses by applying Lean principles and practices", Quality Assurance in Education, Vol. 12 No. 4, pp. 175-187. doi:10.1108/ 0968488041056159.

Fjermestad, J. (2004), "An analysis of communication mode in Group Consensus theorys research", Decision Support Systems, Vol. 37 No. 2, pp. 230-263.

Goldratt, E.M. (1997), Critical Chain, North River Press, Massachusetts, MA, ISBN 0-88427-153-6.

Higgins, D. (2009), "Engaging the small firm in learning: practice based theorizing on complex social knowledge", J Eur Ind Train, Vol. 33 No. 1, pp. 81-96.

Hines, P.A. and Lethbridge, S. (2008), "New development: creating a Lean university", Public Money \& Management, Vol. 28 No. 1, pp. 53-56.

Leitch Review of Skills (2006), "Prosperity for all in the global economy - world class skills - final report", HM Treasury, Westminster, December.

Lin, H.Y., Hsu, P.Y. and Sheen, G.J. (2007), "A fuzzy-based decision-making procedure for data warehouse system selection", Expert Systems with Applications, Vol. 32 No. 3, pp. 939-953.

Radnor, Z. and Bucci, G. (2011), Analysis of Lean Implementation in UK Business Schools and Universities, ISBN 978-0-9567461-1-5, Association of Business Schools Publication, London.

Read, M., Gear, T. and Vince, R. (2012), "Group inquiry to aid organisational learning in enterprises", Journal of the Operational Research Society, Vol. 63 No. 6, pp. 736-747.

Snee, R.D. (2010), "Lean Six Sigma - getting better all the time", International Journal of Lean Six Sigma, Vol. 1 No. 1, pp. 9-29.

Snee, R.D. and Hoerl, R.W. (2002), Leading Six Sigma: A Step-by-Step Guide Based on Experience With GE and Other Six Sigma Companies, ISBN 0-13-008457-3, FT Press, Upper Saddle River, NJ.

The Stationary Office (1992), "Further and Higher Education Act 1992", The Stationery Office Limited, London, ISBN 978-0-11-110166-7.

Vince, R. (2001), "Power and emotion in organisational learning", Hum Relat, Vol. 54 No. 10, pp. 1325-1351.

\section{Further reading}

Bennet, A. and Tomblin, M.S. (2006), "A learning network framework for modern organisations", J Inform Knowledge Management Systems, Vol. 36 No. 3, pp. 289-303.

Shaw, D.C., Eden, C. and Ackermann, D. (2002), "Evaluating Group Consensus theories: improving brainstorming research methodology", Paper No. 2002-2006, Working Paper Series, University of Strathclyde, Glasgow. 